\title{
Developing and implementing the child identification and early intervention flowchart
}

\author{
J odie Ekholm ${ }^{1}$, Paul Hudson ${ }^{2}$, Maree J ohnson ${ }^{3,4}$, Rachel Langdon ${ }^{4}$ \\ 1. Clinical Stream Office, South Western Sydney Local Health District, Sydney, Australia. 2. NSW Clinical Excellence \\ Commission, Sydney, Australia. 3. Faculty of Health Sciences, Australian Catholic University, Sydney, Australia. 4. Centre \\ for Applied Nursing Research, University of Western Sydney/South Western Sydney Local Health District, Sydney, \\ Australia.
}

Correspondence: Rachel Langdon. Address: Centre for Applied Nursing Research, Locked Bag 7103, Liverpool BC NSW 1871, Sydney, Australia. Email: rachel.langdon@sswahs.nsw.gov.au

Received: June 9, 2014

DOI : $10.5430 /$ jnep.v4n11p128
Accepted: July 22, 2014

Online Published: September 22, 2014

URL: http://dx.doi.org/10.5430/jnep.v4n11p128

\section{Abstract}

Background: Early identification of seriously ill children and prompt nursing intervention by nurses within the emergency department (ED) has the potential to improve the health outcomes of children.

Methods: This quality improvement project describes the development of a specific paediatric flowchart, which utilises early warning signs of deterioration, presents age specific paediatric parameters for normal and abnormal signs and symptoms, and uses plotted parameters to enhance early responses from nurses. The implementation process, educational strategies, and refinements of the Child Identification and Early Intervention Flowchart (CHIEIF) are described.

Conclusion: The CHIEIF represents an opportunity for ED nurses to incorporate paediatric specific early warning signs - pain assessment, respiratory effort and work of breathing, and AVPU (Alert, responds to Voice, responds to Pain, Unresponsive) potentially unfamiliar to ED nurses, into daily practice. Education contextualising the use of the CHIEIF into clinical situations is suggested. Further research to evaluate the effectiveness of this flowchart is recommended.

\section{Key words}

Children, Early diagnosis, Early warning signs, Emergency nursing, Nursing education, Nursing observation charts, Paediatric nursing

\section{I ntroduction}

Paediatric patients represent a unique caseload in mixed (adult and paediatric) emergency departments (ED) within Australian hospitals. Within these departments, sick children form a major part of the nurse's caseload. Children manifest a distinct set of critical clinical situations and compensate for altered physiology or pathological processes in defined ways ${ }^{[1]}$. However, children often suffer a rapid deterioration which requires early recognition and response from the emergency nurse. Situations where this response is needed may include instances of hypoxemia secondary to airway obstruction or an acute respiratory disorder, or hypovolaemia due to sepsis, dehydration or rapid fluid shift illness ${ }^{[2,3]}$. Methods that support the early identification and intervention of this deterioration in a seriously ill child in the ED may potentially save the life of the child. Adverse events such as cardiac / respiratory arrest may in part be related to the lack of 
recognition of a deteriorating patient ${ }^{[4]}$. Children are particularly susceptible to deterioration that can easily go undetected as they are often unable to articulate their experiences to nurses or medical officers ${ }^{[5]}$. We undertook this project as a quality improvement initiative for EDs treating both adult and paediatric patients. This paper describes the development and implementation of this specially designed observation chart, known as the Child Identification and Early Intervention Flowchart (CHIEIF), which is aimed at assisting ED nurses to rapidly identify a seriously ill child within their care.

\section{Origins of the criteria in the CHI EI F}

All hospitals use some form of assessment tool to monitor their patients. Assessment tools incorporate early warning signs and other prompts for nurses to take immediate action. These tools may reduce the occurrences of life-threatening adverse outcomes. Alterations in a child's signs and symptoms often herald their deteriorating health status and, as in adults, these changes can form important 'warning signs' which are the basis of the rapid response systems designed for children ${ }^{[5,6]}$. For adults, early signs of clinical deterioration (ES) (e.g., $\mathrm{SpO}_{2}$ 90\%-95\%, SBP 80-100mmHg or 181-240mmHG, Pulse rate 40-49 or 121-140/min, etc), late signs of clinical deterioration (LS) (e.g., $\mathrm{SpO}_{2}<90 \%$, Pulse rate $<40$ or $>140 / \mathrm{min}$, SBP $<80 \mathrm{mmHg}$, GCS $\leq$, etc) and Medical Emergency Team (MET) criteria, discussed below, have all been used and tested extensively ${ }^{[7,8]}$ and have resulted in a small number of criteria predictive of adverse events (e.g., cardiac arrest) ${ }^{[9]}$. The use of early warning systems is now accepted globally, with national and international guidelines being issued by the National Institute of Clinical Excellence ${ }^{[10]}$. Similar principles could be applicable to the use of early warning signs for paediatrics.

An example of this approach is the Physiological Observation Track and Trigger System (POTTS), which combines the charting of physiological observations and the recording of an early warning score (EWS) with the use of colour to indicate where vital signs are abnormal, resulting in the early identification of patients at risk ${ }^{[11]}$. While the POTTS is successful in an adult population, adapting the early warning score for a paediatric population is problematic as the physiological parameters differ depending on the age and development of the child ${ }^{[5]}$.

All facilities within the South Western Sydney Local Health District (SWSLHD), formerly the Sydney South West Area Health Service (SSWAHS), have adopted the MET system, which was first developed by, and implemented at, Liverpool Hospital, NSW. The MET system has been examined through a retrospective analysis of call audit forms, a Death Review database and routinely collected hospital data, with between 34.1 and 37.6 MET calls made per 1000 admissions ${ }^{\text {[12] }}$. Criteria associated with the calls included: being elderly, males, higher Charlson Index [chronic morbidity index], surgical admissions, emergency department admissions, and longer length of stay ${ }^{[12]}$. MET call criteria were defined in the study as systolic pressure $<90 \mathrm{mmg}$, worried, decrease in Glasgow Coma Scale $>2$; Pulse rate $>140$ or $<40$, Respiratory rate $>36$ or $<5$, cardiac arrest, repeated / prolonged seizure, respiratory arrest, or airway threatened ${ }^{[12]}$. However, these criteria are applicable to adults, not paediatric patients.

Early warning systems or criteria for identifying paediatric patients of likely deterioration or adverse events are being developed using similar methodologies to adult systems ${ }^{[1,2]}$. Several vital signs are proposed as being excellent early warning signs in paediatric patients including increases in respiratory rate, heart rate, temperature, and oxygen saturation ${ }^{[1]}$. Tume and Bullock (2004) note that increased respiratory rate is one of the early 'compensatory signs in neonates and small infants, ${ }^{\left({ }^{p .22}\right)}$. Similarly, respiratory disease is a frequent cause of illness in children who present at ED, and cues of severe respiratory distress are key determinants of deterioration in a child ${ }^{[13]}$ including cardiac arrest ${ }^{[14]}$. However, as respiratory rate is inversely proportional to age, with variations of up to 20 breaths per minute from the recommended age rates being observed ${ }^{[13]}$, it cannot be considered in isolation. Rather, it should be considered in conjunction with 'work of breathing' indicators such as nasal flaring, grunting, sternal recession, and see-saw breathing ${ }^{[13,14]}$.

A number of tools, taking into account multiple criteria, have already been developed. The Paediatric Early Warning Score ${ }^{[15]}$ consists of a number of measures, each on a scale of 0 to 3 . The measures include behaviour (playing 
appropriately [0] to lethargy [3]), colour and capillary refill (pink [0] to grey and mottled [3]), and respiratory rate (within normal parameters, no recession or tracheal tug [0] to $>30$ above or 5 below mean normal respiratory rate with sternal recession, tracheal tug or grunting [3] ${ }^{[15]}$. The score for each criteria is added. The total score dictates the action to be taken, for example, an overall score of 4 or more, or a score of 3 on any one measure, indicates deterioration and is a cue for intervention such as increasing the frequency of observations to calling a medical review ${ }^{[15]}$.

Another system, the Paediatric Early Warning System, was tested using a retrospective case (children who had a medical emergency called) / control (other children admitted to the hospital who did not have a medical emergency called) design ${ }^{[16]}$. Indicators for the 16 proposed criteria were examined 1 hour before the medical emergency was called and resulted in $78 \%$ sensitivity (correctly identified a deteriorating child) and $95 \%$ specificity (correctly identifying the child was not deteriorating) and therefore predicting the need for urgent medical attention ${ }^{[16]}$. By using age-specific normal parameters for heart-rate, respiratory rate, and systolic blood pressure, and including general items such as pulse rate, oxygen saturation, capillary refill, level of consciousness, oxygen therapy, bolus fluid and temperature, and calculating the number of medications given within the past 24 hours, an overall evaluation of the status of the child can be formed ${ }^{[16]}$.

Criteria for initiating a medical emergency team for paediatric cardiac arrest have also been reported ${ }^{[2]}$. Examination of the number of deaths and unplanned intensive care unit admissions was undertaken before and after implementation of the MET system — nurse or doctor worried about clinical state, airway threat, hypoxaemia (oxygen saturation $<90 \%$ or $<60 \%$ in any amount of oxygen), severe respiratory distress, apnoea, or cyanosis, and tachypnoea ${ }^{[2]}$. The results indicate the number of cardiac arrests and deaths decreased after the implementation of the MET system, while the number of unplanned intensive care unit admissions increased ${ }^{[2]}$.

NSW Health has also established Clinical Practice Guidelines for the early recognition of a sick child in ED ${ }^{[14]}$. Pivotal clinical indicators to assist health staff to identify a child at risk include the AVPU (Alert, responds to Voice, responds to Pain, Unresponsive), alertness and arousal, breathing including effort, colour and noises, circulation (pulse and capillary refill), fluids in ( $<50 \%$ of normal intake, dry mucous members), fluids out (reduced urine output, vomiting) pain, fever, or rash. For trauma cases, the use of the Airway Breathing Circulation approach is recommended. Hypotension is noted as a very late sign of deterioration in a child, and age specific rates for respiratory and heart rate and systolic blood pressure are provided ${ }^{[14]}$. Most of these aspects have been included in the CHIEIF.

\section{Development of the CHIEIF}

The design, layout and information presented on observation charts influences the recording of observations and the correct interpretation of observations recorded ${ }^{[17]}$. Chatterjee, et al. (2005) created a new "OBS" chart including an early warning scoring system, and reported increases in the detection rates for tachypnoea and hypoxia by $41 \%$ and $45 \%$ respectively. The early warning score was located on the chart and included heart rate, systolic blood pressure, respiratory rate, oxygen saturation and level of consciousness. Several aspects were important from this study and not all parameters were detected similarly. Plotted parameters that are graphically displayed were better detected than written values (80\% plotted versus $58 \%$ written) supporting the use of graphical display of parameters ${ }^{[17]}$. Prompts were also included as they had previously been found to be effective in charts for the resuscitation room ${ }^{[18]}$.

Many children attending the ED experience pain and it is generally recognised that paediatric pain is poorly treated ${ }^{[19]}$. The CHIEIF therefore includes specialised pain assessment tools. The first specialised pain assessment tool included in the CHIEIF uses facial expressions and allows the child to perform a self-assessment of their pain by pointing to a particular expression ${ }^{[20]}$. The other pain assessment tool is a behavioural assessment of the child and is best used for non-verbal patients (children under 8 years of age) or when the self-assessment tool cannot be explained ${ }^{[19]}$. Both tools for the assessment of pain in children have been validated and acknowledged as effective in the assessment of pain ${ }^{[19,20]}$. As emergency nurses have a vital role to play in the early identification of site, intensity of pain as well as managing or 
reducing the pain, the inclusion of a children's pain chart to assess the location and intensity of pain was proposed and included in the CHIEIF.

This quality improvement strategy draws upon the evidence described here and has resulted in the project leaders redesigning an observation chart (see Figure 1). The CHIEIF incorporates 3 major aspects of design: inclusion of mandatory triggers for notification (AVPU) appropriate for children; use of colour where heart rate parameters are abnormal to promote early identification and action by nurses and other health professionals; and the use of graphical representation of vital signs. All elements of existing emergency department flowcharts relevant to children were compared to the proposed CHIEIF and harmonised where appropriate. Similarly, other national and state-wide change processes relating to observation charts were considered and evaluated for effectiveness, including The National Inpatient Medication Chart Policy ${ }^{[21]}$, Health Records and Information Policy ${ }^{[22]}$, SSWAHS Management of Clinical and Non-Clinical Forms ${ }^{[23]}$, and the Final Report of the Special Commission of Inquiry - Acute Care Service in NSW Public Hospitals ${ }^{[24]}$.
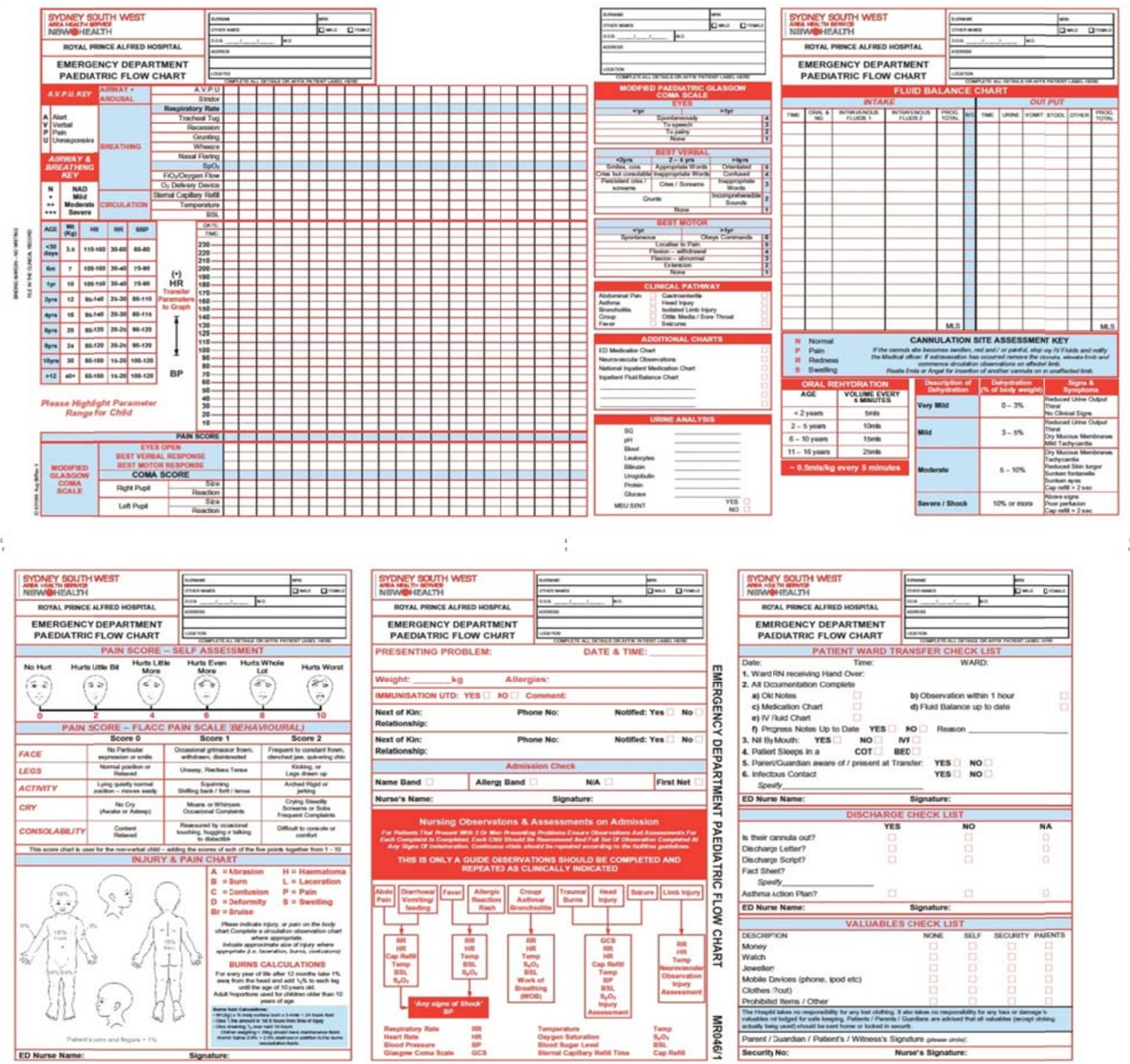

Figure 1. Paediatric Emergency Department Flowchart 


\section{I mplementation process}

\subsection{Design and stakeholder consultation}

The implementation of the CHIEIF followed a similar process to those described elsewhere ${ }^{[25]}$. Key stakeholders in the change process were engaged, key champions or project leaders were identified, and preliminary discussions with clinicians from nursing and medicine were held. An initial flowchart for trialling was developed, with staff education, negotiation with key champions at participating sites, and problem-solving of the introduction process taking place, followed by the stabilisation of the CHIEIF (see Figure 2).

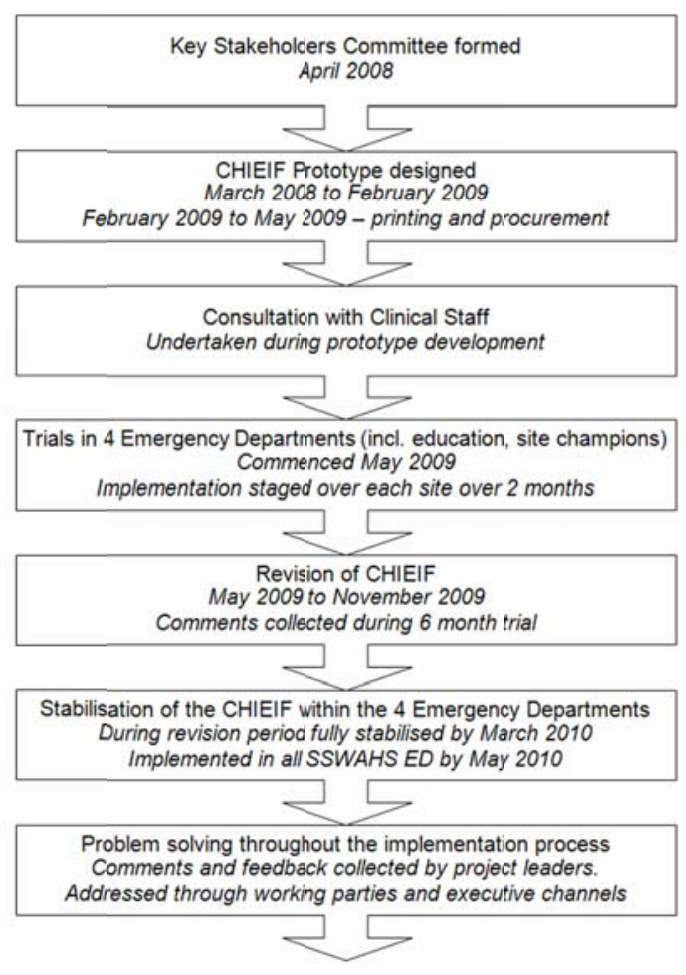

Figure 2. Implementation Process

The SSWAHS Emergency Educator Committee (SEEC), consisting of nursing representatives from each facility including clinical nurse consultants, clinical nurse educators and area the nurse educator, was initially formed in 2005 with the amalgamation of two major area health services.. Representatives from this group were also members of the SSWAHS Emergency Collaborative Group which formed at the same time. This group included nursing representatives from each facility including (in addition to members of the SEEC) nursing unit managers, nurse practitioners and the nursing clinical manager of critical care. It was these two groups who identified the need for a specific form for the paediatric patient, which reflected the various stages of a child's development ${ }^{[5]}$. Previously, a single observation chart was used for all patients within the ED, whether adult or child. The SEEC initially pursued existing emergency observation charts from other departments within NSW and Australia, and identified features that were considered to be useful. Therefore, a new chart with additional functions, including the documenting of vital signs and prompting nurses to take action when signs of deterioration were evident, was created.

A set of different charts for various age groups was initially included on the chart, however, colour coding within the various ages proved too difficult. Staff preferred to highlight the specific parameter for a specific age band, rather than having a different chart for each age group. 
The initial prototype was developed over an 18 month period, and reviewed by clinicians from the SSWAHS Emergency Department Collaborative and the Emergency Education Committee representing stakeholders from all the Emergency Departments within the SSWAHS.

\subsection{CHI EIF in emergency departments}

A trial was conducted in 4 EDs including two large metropolitan trauma centres, one urban facility and one rural facility. Directors of Nursing, in addition to the Clinical Manager, Director for Critical Care Services and SSWAHS Ethics Committee supported and approved the trial.

\section{Learning about the work of breathing}

The implementation process for each ED was undertaken over a six week period. Education sessions, run by Nurse Educators, Clinical Nurse Consultants and Clinical Nurse Educators were provided using a PowerPoint presentation and simulated scenarios. Specifically, these simulated scenarios included video recordings of children suffering from varying states of respiratory distress. These scenarios were used to familiarise nurses with the assessment of respiratory effort or the work of breathing and the recording of respiratory distress on the CHIEIF. Within these sessions, consensus was sought within the groups as to the meaning of the severity levels (Nil, Mild, Moderate or Severe). Staff feedback on the effectiveness of this educational approach was sought through the use of the SEEC website and members of the committee. The feedback given was that this was an effective approach, with staff noting the effectiveness of the use of scenarios with the charts.

\subsection{Final version of the CHI EI F}

The CHIEIF was received with considerable enthusiasm, as staff perceived the tool as better than previous flowcharts in terms of the format and criteria used to identify the deteriorating child. Champions were selected from each ED to facilitate the change process at individual sites and were invaluable over the weekends when educators were not available. While some initial problems occurred as site-specific criteria (on the old form) were not included in the generic child ED flowchart, these were resolved. Some changes to the form were recommended during the trial and embraced, including the addition of free text lines that could be utilised for any additional specific observations that the nurse was required to complete, and having numbers allocated on both sides of the graph. Having a specific paediatric flowchart was also a problem for staff used to having one ED flowchart for both adults and children, as picking up the incorrect form was an issue. Similarly, having charts for varying ages within the child group was problematic and resulted in only one form that incorporated the variations, rather than a series of age-specific CHIEIF forms as initially posed. The final version of the CHIEIF included a coloured stripe to distinguish the adult from the paediatric chart.

The CHIEIF was designed to be commenced within the ED. One of the trial facilities decided to continue the CHIEIF for a period of four hours after a child was admitted to the paediatric ward. This approach was taken in an attempt to improve the transition from the ED to the ward, with staff having the ability to review the observation trends since arrival to the ED rather than a single isolated event. Continual use without problems is now evident throughout the 4 EDs, with the form currently in use within the nine Emergency Department within our health service. The CHIEIF has influenced the development of the NSW Health between the Flags Paediatric Emergency Department Observation Chart. Electronic versions that uphold the guideline principles in early recognition of the deteriorating child have also been developed in conjunction with the paper form. The electronic version will provide specific triggers and alerts that are automatically generated when an early or late sign of deterioration is recorded.

\section{Discussion}

The early identification of a child who is deteriorating within an ED is a clinical practice problem that has challenged emergency nurses. The development of a paediatric specific flowchart was seen as a must within the SSWAHS ED community as the previously used parameters were not appropriate for the paediatric patients within their EDs. This challenge was acknowledged by the staff at our local EDs and a strategy to enhance nurses' abilities to correctly and 
promptly identify a child in jeopardy was addressed. A specific observation chart which incorporated important evidence-based triggers or early warning signs originally designed for adults ${ }^{[11]}$ and later applied to children ${ }^{[15,16]}$ was developed. Other aspects of importance such as appropriate tools to assess pain location and intensity ${ }^{[19,20]}$ were also included. The importance of the 'work of breathing' as an indicator of change in a child's health status has, from anecdotal comments, assisted nurses to identify and respond. The use of age-appropriate parameters, and formatting aspects of graphical presentation of data, colour coding, and distinguishing markings for adults and paediatric charts were central to the design of the CHIEIF.

As in any other change process, the use of a structured approach inclusive of key stakeholders from the commencement of the change process has been successfully used with positive outcomes. Engagement of educators and managers, who were alerted to the problem, has resulted in a sustainable change in the observation of children and clinical decision making processes of ED nurses. Utilising champions within each facility promoted acceptance within the departments. Clinical Nurse Consultants and Clinical Nurse Educators, as stakeholders, facilitated the implementation of the chart within their own facility. These facility stakeholders were pivotal to continual bedside education and the auditing the use of the CHIEIF following implementation.

Education programs using simulations and scenarios with charting of the 'work of breathing' and other aspects have supported nurses to grasp this key sign and symptom in children's physiology and pathophysiology. The CHIEIF is now an accepted observation tool that assists nurses to promptly identify the deteriorating child. The implementation process of the CHIEIF was a lengthy process from the initiation of the project to the stabilisation of the form within the busy EDs taking some 2.5 years. Integration of the CHIEIF observation chart is occurring through its inclusion within Emergency Nursing courses within our health service.

The CHIEIF, like any other assessment form, as a tool in isolation would have limited effectiveness. Education in identifying the subtle signs of deterioration has been essential. EDs would benefit from the introduction of tools such as the CHIEIF through utilising simulated scenarios based on real events to highlight how the CHIEIF can impact patient care, facilitate early identification of deterioration and support staff decision making. Electronic recording of vital signs is a logical step forward and may emerge in the future. An electronic module of the CHIEIF would be beneficial if it does not detract from the need for ED nurses to be at the child's bedside. Further research, using such outcomes as medical emergency team calls, frequency of reporting children for medical review and other measures could be used to evaluate the effectiveness of the CHIEIF.

\section{Conclusion}

Clinicians at the front line of our EDs aim to improve a child's illness or save a child's life by prompt intervention. The CHIEIF provides a valuable tool for the early identification of a deteriorating child for ED nurses, particularly those with limited experience. The importance of supportive education that contextualises the tool and its value within clinical practice is emphasised. Stakeholder engagement and the use of local champions have supported the change process. Paediatric flowcharts such as the CHIEIF should be included in Emergency Nursing courses. Anecdotal evidence to date, suggests that this tool prompts and supports action by the nurse in the 'escalation' of the paediatric patient's care. The use of known early warning signs criteria (colour and graphic representation of signs and symptoms beyond normal parameters) has anecdotally resulted in the early recognition of a deteriorating child and subsequent call for medical review. Electronic modules of the CHIEIF may prove useful in the future. Further research is planned by this team to evaluate the outcomes of this quality improvement in emergency nursing care.

\section{References}

[1] Tume L, Bullock I. Early warning tools to identify children at risk of deterioration: A discussion. Paediatric Nursing. 2004; 16(8): 20-3. http://dx.doi.org/10.7748/paed2004.10.16.8.20.c943. 
[2] Tibballs J, Kinney S, Duke T, Oakley E, Hennessy M. Reduction of paediatric in-patient cardiac arrest and death with a medical emergency team: Preliminary results. Archives of Disease in Childhood. 2005; 90(11): 1148-52. http://dx.doi.org/10.1136/adc.2004.069401.

[3] Winberg H, Nilsson K, Aneman A. Paediatric Rapid Response Systems: A literature review. Acta Anaesthesiologica Scandinavica. 2008; 52(7): 890-6. http://dx.doi.org/10.1111/j.1399-6576.2008.01672.x.

[4] Tippins E. How emergency department nurses identify and respond to critical illness. Emergency Nurse. 2005; 13(3): 24-33. http://dx.doi.org/10.7748/en2005.06.13.3.24.c1048.

[5] Haines C, Perrott M, Weir P. Promoting care for acutely ill children: Development and evaluation of a paediatric early warning tool. Intensive and Critical Care Nursing. 2006; 22(2): 73-81. http://dx.doi.org/10.1016/j.iccn.2005.09.003.

[6] Edwards E, Powell C, Mason B, Oliver A. Prospective cohort study to test the predictability of the Cardiff and Vale paediatric early warning system. Archives of Disease in Childhood. 2009; 94(8): 602-6. http://dx.doi.org/10.1136/adc.2008.142026.

[7] Harrison G, Jacques T, McLaws M, Kilborn G. Combinations of early signs of critical illness predict in-hospital death: The SOCCER study (signs of critical conditions and emergency responses). Resuscitation. 2006; 71(3): 327-34. http://dx.doi.org/10.1016/j.resuscitation.2006.05.008. PubMed PMID: 17069957.

[8] Harrison G, Jacques T, Kilborn G, McLaws M. The prevalence of recordings of the signs of critical conditions and emergency responses in hospital wards: The SOCCER study. Resuscitation. 2005; 65(2): 149-57.

http://dx.doi.org/10.1016/j.resuscitation.2004.11.017.

[9] Jacques T, Harrison G, McLaws M, Kilborn G. Signs of critical conditions and emergency responses (SOCCER): A model for predicting adverse events in the inpatient setting. Resuscitation. 2006; 69(2): 175-83. http://dx.doi.org/10.1016/j.resuscitation.2005.08.015. PubMed PMID: 16497427.

[10] Cuthbertson B. Optimising early warning scoring systems. Resuscitation. 2008; 77(2): 153-4. http://dx.doi.org/10.1016/j.resuscitation.2008.03.013.

[11] Oakey R, Slade V. Physiological observation track and trigger system. Nursing Standard. 2006; 20(27): $48-54$. http://dx.doi.org/10.7748/ns2006.03.20.27.48.c4094.

[12] Young L, Donald M, Parr M, Hillman K. The Medical Emergency Team system: A two hospital comparison. Resuscitation. 2008; 77(2): 180-8. http://dx.doi.org/10.1016/j.resuscitation.2007.11.016.

[13] Aylott M. Observing the sick child: Part 2a. Respiratory assessment. Paediatric Nursing. 2006; 18(9): 38-44. http://dx.doi.org/10.7748/paed2006.11.18.9.38.c8321.

[14] NSW Department of Health. Recognition of a Sick Baby or Child in the Emergency Department 2011 [cited 2011]. Available from: http://www.health.nsw.gov.au/policies/pd/2011/pdf/PD2011_038.pdf.

[15] Monaghan A. Detecting and managing deterioration in children. Paediatric Nursing. 2005; 17(1): 32-5. http://dx.doi.org/10.7748/paed2005.02.17.1.32.c964.

[16] Duncan H, Hutchison J, Parshuram C. The Pediatric Early Warning System score: A severity of illness score to predict urgent medical need in hospitalized children. Journal of Critical Care. 2006; 21(3): 271-8. http://dx.doi.org/10.1016/j.jcrc.2006.06.007.

[17] Chatterjee M, Moon J, Murphy R, McCrea D. The "OBS" chart: An evidence based approach to re-design of the patient observation chart in a district general hospital setting. Postgraduate Medical Journal. 2005; 81(960): 663-6. PMid:16210466 http://dx.doi.org/10.1136/pgmj.2004.031872

[18] Knight S, Calvesbert K, Clarke J, Williamson J. Developing a nursing observation chart. Emergency Nurse. 2002; 10(3): 16-7. http://dx.doi.org/10.7748/en2002.06.10.3.16.c1062.

[19] Merkel S, Voepel-Lewis T, Shayevitz J, Malviya S. The FLACC: A behavioral scale for scoring postoperative pain in young children. Pediatric Nurse. 1997; 23(3): 293-7. PMid:9220806

[20] Wong D, Baker C. Pain in children: Comparison of assessment scales. Pediatric Nursing. 1988; 14(1): 9-17. PMid:3344163

[21] NSW Department of Health. Medication Chart: NSW Implementation of the National Inpatient Medication Chart 2006. Available from: http://www.health.nsw.gov.au/policies/pd/2006/pdf/PD2006_028.pdf.

[22] Corporate Governance and Risk Management. The Patient Matters Manual. Chapter 9: Health Records and Information 2008. Available from: http://www.health.nsw.gov.au/resources/policies/manuals/pdf/pmm_9.pdf.

[23] Sydney South West Area Health Service. Management of Clinical and Non-Clinical Forms 2010. Available from: http://intranet.sswahs.nsw.gov.au/SSWpolicies/pdf/SSW_PD2010_014.pdf.

[24] Garling P. Final Report of the Special Commission of Inquiry into Acute Care Services in NSW Public Hospitals 2008 [14 Jul 2009]. Available from: http://www.lawlink.nsw.gov.au/Lawlink/Corporate/ll_corporate.nsf/vwFiles/E_Overview.pdf/\$file/E_Overview.pdf.

[25] Sellitto C, Carbone D. Success factors associated with Health Information Systems implementation: A study of an Australian regional hospital. Journal of Business Systems, Governance and Ethics. 2007; 2(4): 39-54. 